\title{
Effects of aging in retinal image quality
}

\author{
Pablo Artal \\ Instituto de Optica, Consejo Superior de Investigaciones Científicas, Serrano 121, 28006 Madrid, Spain
}

Manuel Ferro and Ismael Miranda

Departamento de Oftalmología, Hospital Doce de Octubre, 28041 Madrid, Spain

\section{Rafael Navarro}

Instituto de Optica, Consejo Superior de Investigaciones Científicas, Serrano 121, 28006 Madrid, Spain

Received July 22, 1992; revised manuscript received November 25, 1992; accepted January 11, 1993

\begin{abstract}
The retinal image quality characterized by the modulation-transfer function of the eye was measured for two groups of subjects aged in the late twenties and mid sixties, respectively. In both groups, we obtained modulation transfer functions by using a double-pass method under the same experimental conditions: 4-mm artificial pupil, paralyzed accommodation, and objective control of the refractive state and centering. Results showed lower values of modulation in the retinal image for older subjects compared with the younger subjects. The modulation transfer function ratio is similar to that previously found for contrast-sensitivity measurements with subjects in the same age groups. These results suggest that a significant fraction of the loss in spatial vision with age has an optical origin. Apart from the well-known increase in intraocular scattering, there also appears to be an increment in ocular aberration that causes an additional reduction in the contrast of retinal images.
\end{abstract}

\section{INTRODUCTION}

The effects of aging on the human visual system have been widely studied in recent years. Various authors have shown that many aspects of visual performance, and spatial vision in particular, deteriorate with age (see Refs. 1-3 for general reviews). To evaluate the losses of spatial visual performance with age, traditionally mainly visual acuity ${ }^{4}$ and more recently the contrast-sensitivity function $(\mathrm{CSF})^{5,6}$ have been measured. Although results reported by different authors are somewhat contradictory, probably because of the different experimental procedures used, it is generally accepted that aging reduces contrast sensitivity mainly at intermediate and high spatial frequencies. Nevertheless, there is not general agreement regarding either who should be considered a normal old subject ${ }^{7}$ or the finding of a general, standard procedure for contrast-sensitivity measurements. ${ }^{8}$ In addition, optical factors such as pupil size, accommodation, and refractive state have the potential to introduce variability in contrast sensitivity, although recent studies conducted with an accurate control of these factors ${ }^{6,9}$ have provided strong evidence for a decline in contrast sensitivity with age. Both optical and neural factors must play some role in the reduction of spatial visual performance with age, but their relative contributions are not well known. To date, various different approaches have been used to evaluate these relative contributions. One method consists of determining both the coherent CSF, by using interference fringes on the retina and bypassing the eye's optics, ${ }^{10,11}$ and the overall incoherent CSF. Although this kind of interferometric technique when applied to older subjects yielded contradictory results, ${ }^{12,13}$ a recent study ${ }^{14}$ measuring contrast sensitivity for interference fringes concluded that neural changes have a small effect in CSF deterioration with age. Another procedure is to compare CSF's for older normal subjects with those for aged patients who have had intraocular lenses (IOL's) implanted after cataract surgery. ${ }^{15}$ In principle, this would permit one to bypass the effect of the old crystalline lens, which has been proposed as a major cause of the age-related reduction in contrast sensitivity. If this is true, older subjects with implanted IOL's should have a better CSF than do older normal subjects, and the relative influence of the old lens could be determined by comparison of both CSF's. However, the CSF's for these two groups of subjects ${ }^{15}$ were approximately the same.

Instead, we report here the results of a direct approach for assessing the relative contribution of the eye's optics to the CSF decline with age by objectively measuring the modulation-transfer function (MTF) of the eye for two age groups, younger and older subjects, and by comparing these results with contrast-sensitivity measurements. Although most of the optical-related changes in the aging eye, e.g., absorption of light in the optical media, intraocular scattering, senile miosis, and reduction in the accommodative range, have been well studied in the past, little research has been conducted on the effects of aging on the MTF or optical aberrations and their influence on contrast sensitivity.

In the present study we used a double-pass system to measure the retinal image quality in older subjects (midsixties) and compared those with the results obtained for younger subjects (late twenties). The experimental system was previously developed in our laboratory, ${ }^{16}$ and it was applied to assess the retinal image quality of the eye in the fovea and periphery, ${ }^{17}$ except always in young observers. Our double-pass method has practical advan- 


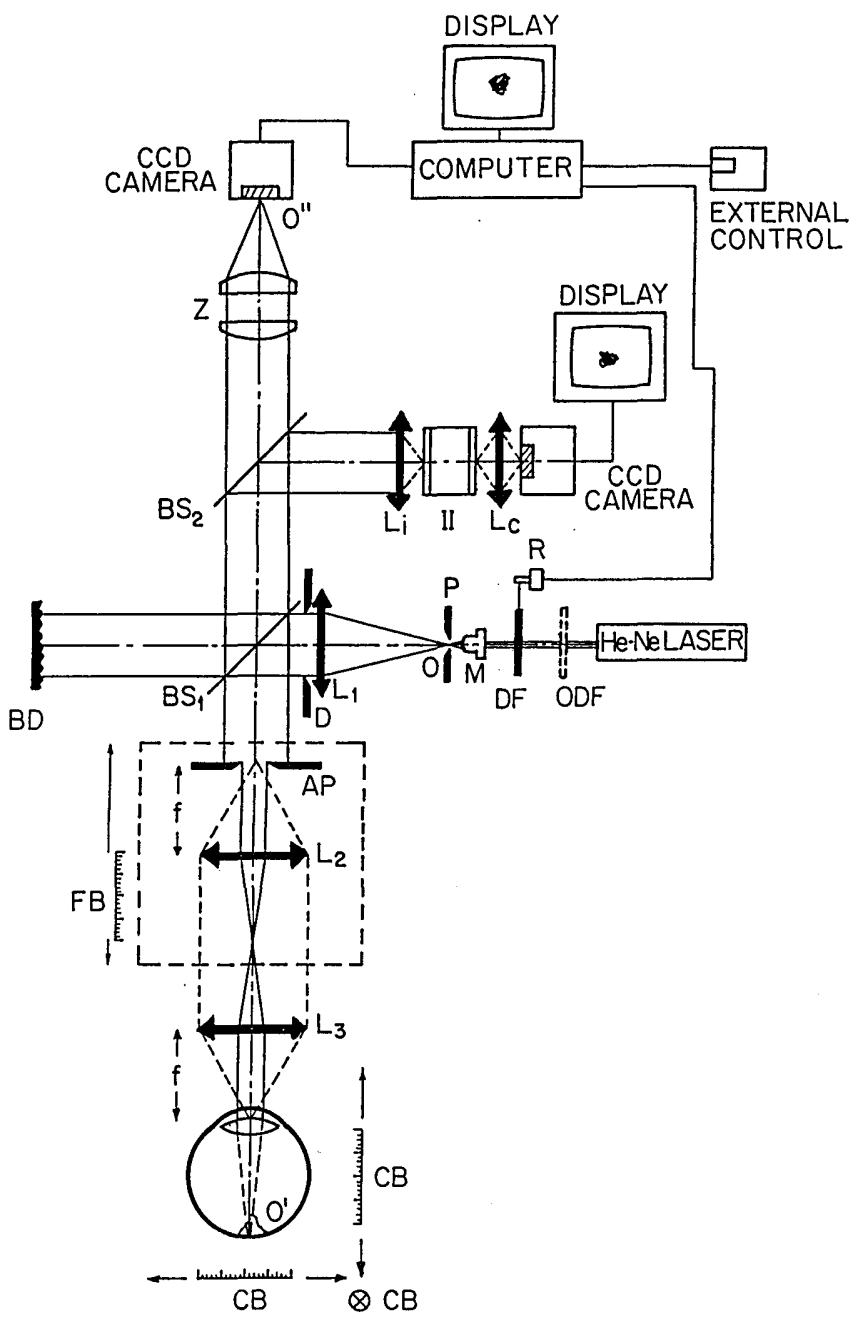

Fig. 1. Experimental setup. AP, artificial pupil (4-mm spot); $\mathrm{BS}$, pellicle beam splitter; $\mathrm{BD}$, light trap; $\mathrm{CB}$, centering micrometric stage; D, spot; DF, high-density ND filter (ND = 1.6); FB, focusing block, II, image intensifier; $L$, lens; $L_{i}$, lens forming the aerial retinal image on the II; $\mathrm{L}_{c}$, lens forming the II-screen image on a second CCD camera; $M$, microscope objective; $O$, point object; ODF, optional ND filter (ND $=0.2-1.6)$; $\mathrm{P}$, pinhole; $\mathrm{R}$, rotary solenoid (shutter); $\mathrm{Z}$, zoom lens.

tages for this kind of study-mainly the subject's comfort and procedural rapidity-permitting us to obtain measurements in untrained naïve subjects. Moreover, we recently evaluated the reliability of the method by performing several experiments. In one of these experiments we studied the influence of the retinal reflection on the double-pass MTF measurements by recording simultaneously the retinal image of two points separated by $1 \mathrm{deg}$ in the fovea. ${ }^{18}$ Despite the difference in retinal thickness at these locations, both aerial images had approximately the same spread. This result indicates a relatively small effect of retinal reflection and lends additional support to the validity of the double-pass method to estimate the eye's MTF in most practical cases. On the other hand, since this particular study requires that the experimenter have careful control of different optical factors affecting image quality during the experiment, we modified the experimental setup, including an additional system to control objectively the refractive state and centering of the subjects, by monitoring the aerial image in real time during the MTF measurements.

\section{DOUBLE-PASS SYSTEM AND EXPERIMENTAL PROCEDURE}

The basic double-pass system has been described in detail elsewhere. ${ }^{16-18}$ It consists of two parts: the recording of short-exposure aerial images of a point object after a double pass through the optical media of the eye and digital image processing, including averaging of aerial images, Fourier transform, and computation of the square root to obtain the single-pass MTF.

Figure 1 shows a diagram of the experimental system. The beam emerging from a He-Ne laser (nominal power $10 \mathrm{~mW}$ ) first passes through an optional neutral-density (ND) filter ( $\mathrm{ND}=0.2-1.6$ ), which is used to attenuate and adjust the light intensity on the CCD camera to the optimum range. A second high-density filter $(\mathrm{ND}=1.6)$ is mounted on a rotary solenoid, so it can be moved in and out of the beam. The attenuation of this filter was chosen to permit the subject to use the point object, $\mathrm{O}$, as a comfortable fixation target and also to have enough energy to monitor in real time the aerial image with the imageintensifier camera system that is used for control purposes. This high-density ND filter is removed and is synchronized with the camera, during the short exposure time, typically $100 \mathrm{~ms}$, acting as a flash shutter. The beam is spatially filtered by a $20 \times$ microscope objective and a $10-\mu \mathrm{m}$ pinhole, which acts as our point object. The emerging beam is collimated by lens $L_{1}\left(f^{\prime}=200 \mathrm{~mm}\right)$; $\sim 8 \%$ of the light is reflected toward the eye by a pellicle beam splitter, and the light that is transmitted is removed from the beam path by a light trap. Before entering the eye, the beam passes through an afocal system consisting of two equal lenses, $\mathrm{L}_{2}$ and $\mathrm{L}_{3}\left(f^{\prime}=120 \mathrm{~mm}\right)$. This permits the use of an artificial pupil by imaging the spot on the subject's pupil plane by using lenses $L_{2}$ and $L_{3}$, independently of their relative position. This provides a method to modify the subject's refractive state continuously by moving the focusing block backward and forward. The eye forms the image of point $\mathrm{O}$ on retina $\mathrm{O}^{\prime}$, and a small fraction of the light is reflected back, passing again through the optical media of the eye (second pass), lenses $\mathrm{L}_{2}$ and $\mathrm{L}_{3}$, and the beam splitters. Finally the zoom lens $\left(f_{z}{ }^{\prime}=60-300 \mathrm{~mm}\right)$ forms aerial images $\mathrm{O}^{\prime \prime}$ on a CCD camera. The camera (Hitachi KP-140), along with the frame grabber (Matrox MVP-AT), was previously calibrated.

\section{Real-Time Monitoring of the Aerial Image}

To minimize the experimental variability in the measurements, it is necessary to record the aerial images under controlled conditions of refractive state and centering. This is achieved with a control subsystem in the experimental setup to monitor the aerial image of a point test in real time. This second subsystem is based on the method of Arnulf et al. ${ }^{19}$ for the dynamic recording of retinal images. It consists of a second pellicle beam splitter that reflects the light returning from the eye; a lens $\mathrm{L}_{i}$ forms the aerial retinal image on an image intensifier, and another lens $L_{c}$ forms the image of the image-intensifier screen on a second CCD camera. This permits monitoring of the retinal aerial image in real time comfortably and under safe conditions for the subject. This system provides an objective control of the refractive state and centering during the recording of retinal images. These two similar systems must be used simultaneously because, 
although the image-intensifier system is useful for control, only the main system permits linear recording of shortexposure images for the subsequent MTF calculations.

\section{Experimental Procedure and Image Processing}

The measurements are obtained with monochromatic light $(632 \mathrm{~nm})$, with only spherical refractive compensation adjusted by placement of the focusing block (FB) in the desired position. Point source $O$ was also used as the fixation target, so the resulting MTF's correspond to the fovea. During the snapshots (100-ms exposure time, with an $\sim 15$-s interval between snapshots), the high-density ND filter is removed from the beam, and the maximum laser irradiance in the pupil plane is of the order of $0.3 \mathrm{~mW} / \mathrm{cm}^{2}$, which means that for a $4-\mathrm{mm}$ pupil diameter the laser power entering the eye is $<0.04 \mathrm{~mW}$. This is a worst-case value for older subjects who have poor retinal image quality, which widely spread the intensity of the aerial image. For younger emmetropic subjects who have a good image quality, the beam intensity typically is further reduced by 1 to $2 \log$ units. Since the exposure time is of the order $100 \mathrm{~ms}$, our maximum output energy is $\sim 1 / 50$ or less of the maximum limit permitted by American National Standards Institute safety standards. ${ }^{20}$

The short-exposure aerial images are averged to remove speckle noise to simulate incoherent imaging conditions. It was previously shown ${ }^{16}$ that, to remove speckle noise, it is necessary to average 32 or more short-exposure images to obtain an acceptable signal-to-noise ratio. In this particular study, since we considered that high accuracy is of less importance than are rapidity of the measurements and subjects' comfort, we computed each MTF from the average of only 16 frames. The actual procedure is to take two series of eight exposures each and compute the average afterward. Each snapshot is delivered by the experimenter when the desired conditions of centering and focus are reached. The resulting image is accepted only when it is free of artifacts from blinks or other causes and when the intensity is within a useful range. Nevertheless, the relative number of accepted images is quite high, which guarantees the rapidity of the procedure. After an image-processing window of $256 \times$ 256 pixels is set, a background image, obtained by placing a black diffuser in the pupil plane instead of the eye, is subtracted from the aerial image. We subsequently average the short-exposure retinal images, and we remove the remaining background by subtracting the average intensity value computed in the four corners of the image. The Fourier transform of the aerial image is computed, and ultimately we obtain the single-pass MTF as the square root of its modulus. The experimental procedure is fast, rather easy, and comfortable for the subjects. A complete MTF measurement typically takes $\sim 15 \mathrm{~min}$ on average, including subject's centering, the collection of 16 frames, and processing.

\section{Control of Optical Factors During the Experiment}

\section{Paralyzed Accommodation}

Because of the large differences in the accommodative response between younger and older subjects, the measurements in both groups were performed with paralyzed accommodation. Three drops of cyclopentolate $1 \%$ were instilled in the subject's eye, with a waiting period of $5 \mathrm{~min}$ between drops; measurements were begun 30 min after the final drop was instilled.

\section{Artificial Pupil}

The effect of pupil size in the aerial retinal images is of major importance. ${ }^{21}$ In this particular case systematic differences in pupil size were found under the same conditions of luminance between our two groups of subjects because of senile miosis. To avoid this variability we performed all measurements with an artificial pupil of 4-mm diameter.

\section{Centering}

The use of artificial pupils imposes the need for careful control of centering during the experiment. It has been shown that a small decentering of the pupil introduces significant astigmatism that degrades the retinal image quality. ${ }^{22}$ The subject's head is fixed by a bite bar, which is mounted on a micrometric positioner and is used to align the observer in the three spatial directions with respect to the artificial pupil. Control of centering is achieved by monitoring the retinal image, assuming that the astigmatism-free retinal image corresponds to the best-centered pupil. During the entire process the subject is continuously recentered to the optimum position.

\section{Refractive State}

The refractive state factor introduces a large variability in any kind of spatial-vision measurement, including both optical MTF and contrast sensitivity. Here we objectively control this variability by moving the focusing block and looking for the best (smallest and most symmetrical) aerial image, which is monitorized by the control subsystem. We determine the best correction by using this method before collecting images and maintain it throughout the measurements.

\section{Selection and Clinical Evaluation of Subjects}

The results were obtained in two groups of five subjects: younger subjects, with mean age of 29 years (2 standard deviations), and older subjects, with mean age of 64 years (3 standard deviations). The subjects were selected after they passed a standard ophthalmological test; we accepted for this study only those subjects with visual acuity 1 $(20 / 20)$ or better, with a spherical correction between -2 and +2 diopters (D) and $<0.25 \mathrm{D}$ of astigmatism. The selected subjects had normal visual fields, anterior chambers after slip-lamp examination, fundi after indirect ophthalmoscope exam, color vision, and intraocular pressures. We also performed a clincial evaluation of the CSF for every subject, using the Vectorvision CVS-1000 chart.

\section{EXPERIMENTAL RESULTS}

\section{Comparison of Aerial Retinal images and MTF's for Younger and Older Subjects}

Figure 2 shows four typical examples of aerial retinal images of a point test for two younger [Fig. 2(a)] and two older [Fig. 2(b)] subjects. The axis, in minutes of arc, is 

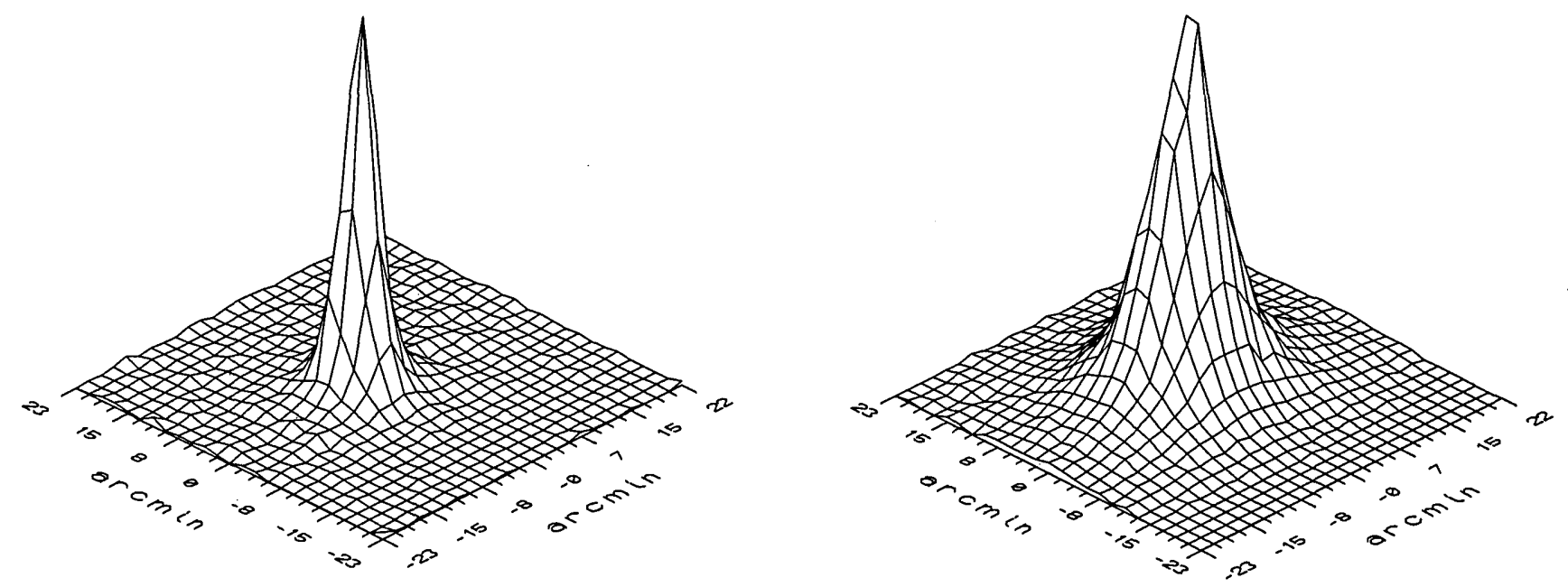

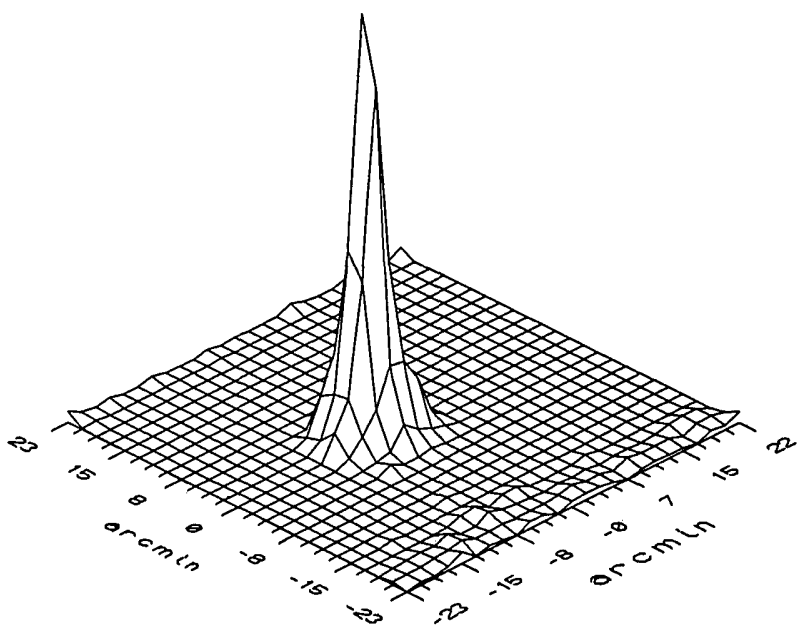

(a)

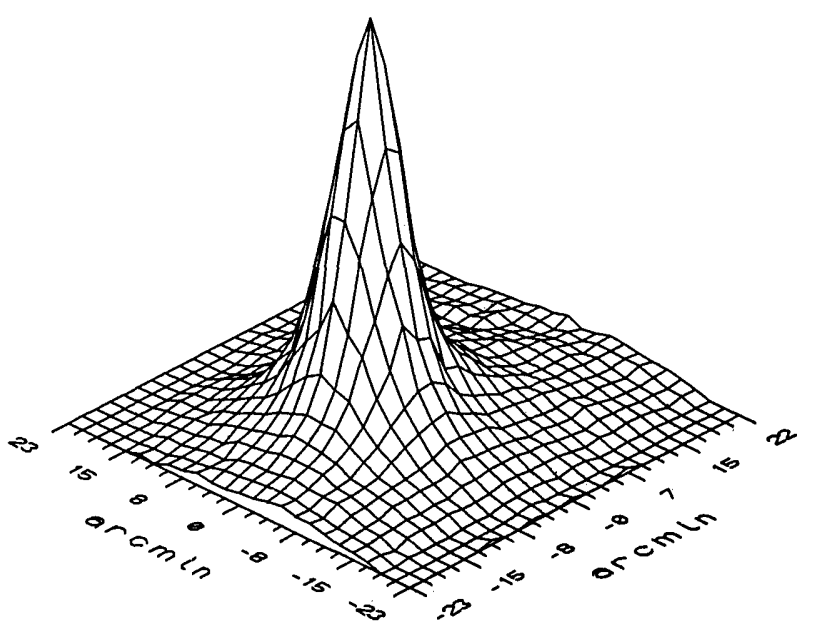

(b)

Fig. 2. Aerial retinal images for four subjects: (a) younger subjects, (b) older subjects. The $x-y$ axis is in minutes of arc.

the same for the four three-dimensional plots, permitting a direct comparison. It must be noted that, although in both cases the results were obtained under the same experimental conditions, there are significant differences in the spread of the images. Since aerial images are affected twice by the aberrations because of the double pass through the eye, to obtain a quantitative comparison we computed the single-pass MTF according to the procedure outlined above. The mean MTF for each group was computed, and, finally, since our observers are practically astigmatism free, an averaged one-dimensional MTF was calculated as the radial projection (averaging over all orientations) of the two-dimensional MTF. This permits an easier comparison not only between the MTF's of the two groups but also with contrast-sensitivity measurements. Figure 3 presents the one-dimensional radial projections of the MTF's for younger and older subjects, together with error bars (standard error of the mean), showing the variability of retinal image quality between subjects. It should be noted that this variability in the results is much smaller than are the differences between the mean MTF's for each group. Although the error bars are larger for the group of younger subjects, the relative variability (standard error over modulation value) is similar for both groups.

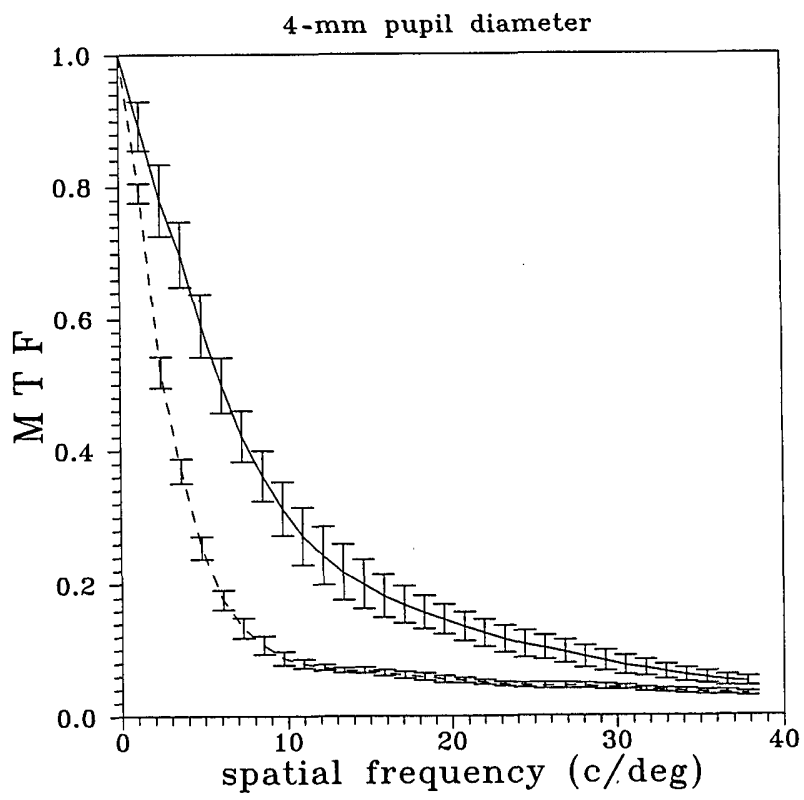

Fig. 3. Averaged one-dimensional radial profiles of the MTF's for younger subjects (mean age 29 years; solid curve) and older subjects (mean age 64 years; dashed curve). Error bars indicate the standard error of the mean to show the variability of the results. 


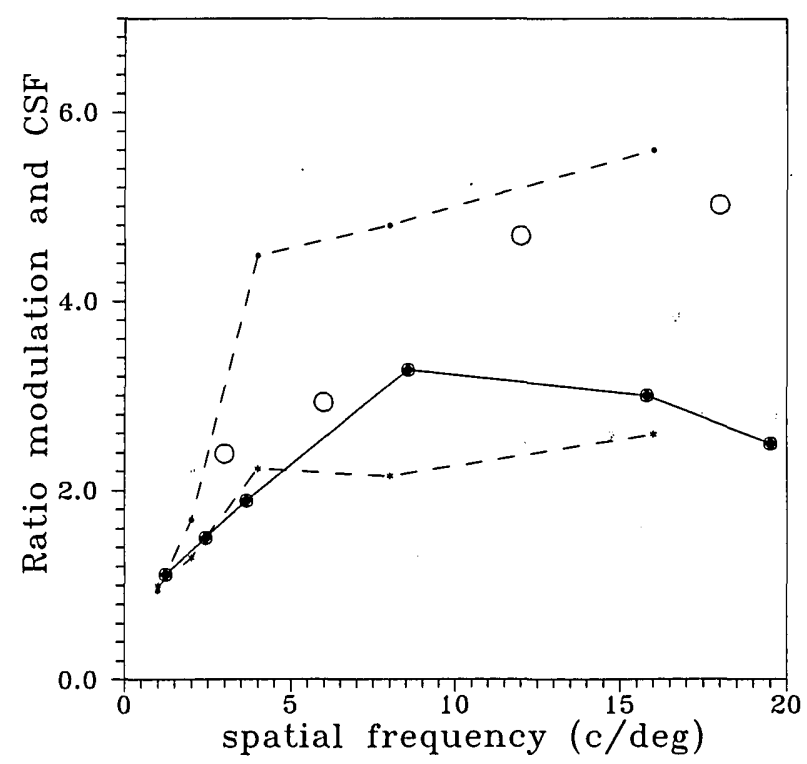

Fig. 4. Ratios of CSF's and MTF's for groups of younger and older subjects: MTF ratio (solid curve and filled circles), clinical CSF ratios for the same subjects (open circles), and CSF ratios from Owsley et al. ${ }^{6}$ (dashed curve).

\section{Ratios between Younger and Older Subjects' MTF's and CSF's}

To evaluate the relative effect of the optical degradation for older subjects, we calculated the ratio between younger and older subjects' MTF's for different spatial frequencies. This ratio is compared with the CSF ratio previously reported by Owsley et al. ${ }^{6}$ and with that obtained in our CSF clinical evaluation in the same observers in which MTF's were measured. Figure 4 summarizes all these results: the MTF ratio is $\sim 2$ for spatial frequencies near 5 cycles per degree (c/deg), increasing to $\sim 3$ for larger spatial frequencies. This ratio is qualitatively similar to that of the CSF's as reported by Owsley et al. ${ }^{6}$ when comparing observers aged in their twenties and sixties. The open circles are the ratios of the clinical CSF's that we obtained for the same observers. At higher spatial frequencies, there are larger differences between subject groups in the CSF data than in the MTF data, probably because senile miosis caused an additional reduction in the older subjects' CSF's, since those CSF data were collected with natural pupil. It must be noted that we cannot use these ratios for a direct comparison because the CSF's of Owsley et $a l^{6}{ }^{6}$ were obtained in another set of subjects and in white light, whereas our MTF results are monochromatic; on the other hand, our clinical CSF data, although corresponding to the same subjects, were also collected in white light and without a precise control of pupil size and accommodation. Nevertheless, based on the analysis of these results, we can suggest that an important part of the contrast-sensitivity degradation is explained by the larger amount of ocular aberrations found in normal older eyes, which cause a lower MTF and consequently a loss in contrast sensitivity.

\section{Normal Older Subjects versus Subjects Implanted with IOL's}

Optical density and scattering in the lens increase with age. As is mentioned above, an indirect method of com- paring the relative influence of the lens in the contrastsensitivity losses has been based on the comparison of normal older subjects with those implanted with IOL's. In a recent study ${ }^{23}$ we also used the double-pass method to measure the ccular MTF in patients with implanted monofocal and three different types of bifocal IOL's. The MTF's for eyes implanted with bifocal IOL's were lower by a factor of 2 than those with monofocal IOLs, although a similar resolution was maintained. From these data we selected the mean MTF for five subjects who were implanted with monfocal IOL's to be compared with the mean MTF obtained for the group of normal older subjects (Fig. 5). We found both MTF's to be quite similar and in agreement with previously reported CSF data for two similar groups of subjects. ${ }^{15}$

\section{Comparison of Younger and Older Subjects Implanted with IOL's}

An additional, interesting experiment is to compare the eye's optical performance obtained by our double-pass method in younger (aged 30-40 years) and older (aged 6070 years) subjects implanted with the same type of monofocal IOL's. Figure 6 shows a three-dimensional plot of the aerial retinal images for a young subject, MG (age 32 years), for his right eye (normal) and his left eye (implanted with a monofocal IOL). Figure 7 presents the one-dimensional radial-projection MTF's for subject MG's right eye and left eye, and the mean MTF for older subjects implanted with monofocal IOLs. MTF's for both younger and older subjects implanted with IOL's are also similar, which has several implications that are discussed next.

\section{DISCUSSION}

The MTF results of ten normal subjects, five younger $(\sim 29$ years old) and five older ( $\sim 64$ years old) show a clear deterioration of retinal image quality with age. These

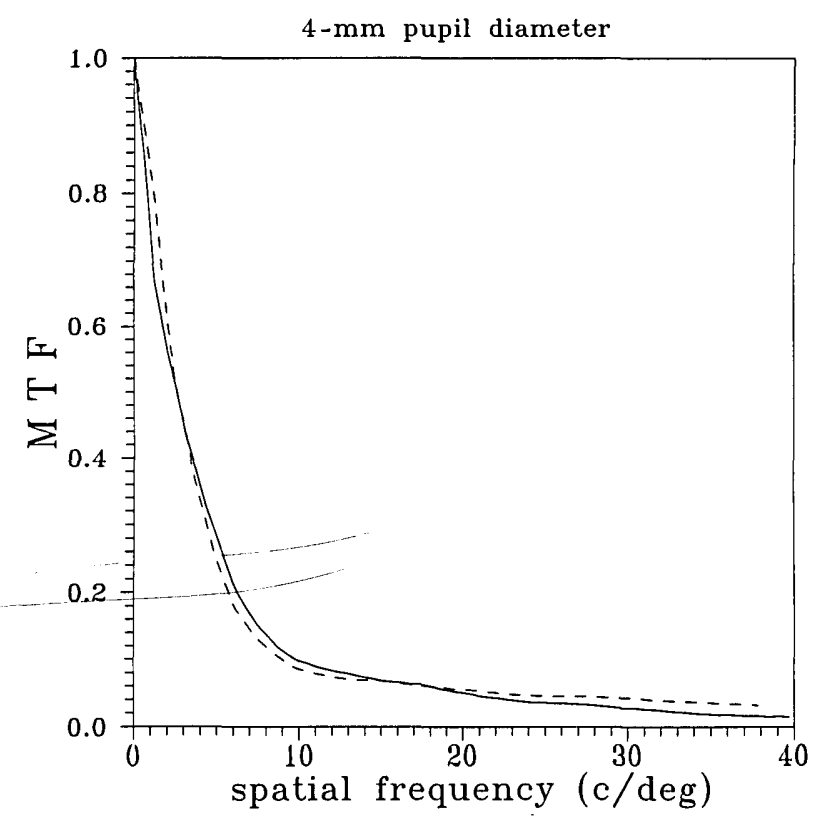

Fig. 5. Comparison of mean M'TF's obtained for the older group (mean age 64 years; dashed curve) and for older patients (five subjects) implanted with monofocal IOL's (solid curve). 


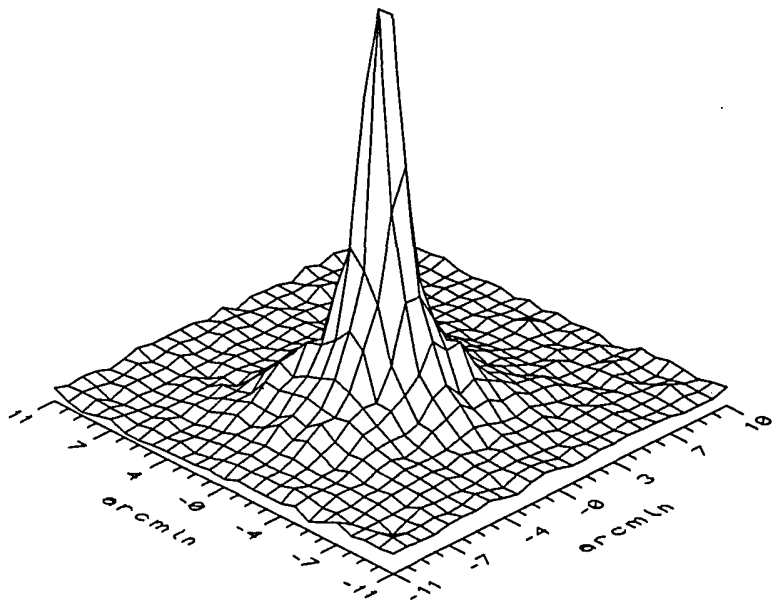

(a)

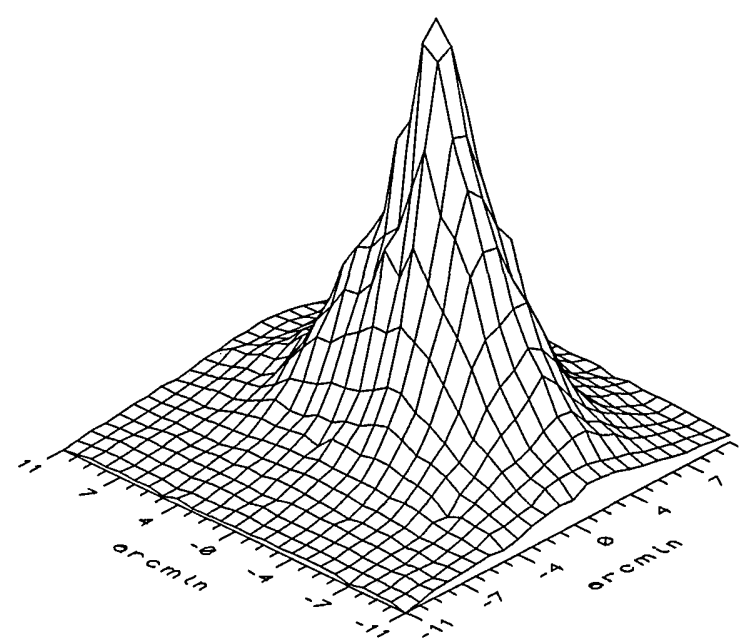

(b)

Fig. 6. Retinal aerial images for a young subject (MG) (32 years old). (a) His left eye with an implanted monofocal IOL. (b) His right, normal eye. The scale is the same in both graphs (minutes of arc) to permit a direct comparison.

measurements were obtained with accurate control of the most significant optical factors that are likely to introduce variability in retinal image quality. The differences between age groups should actually correspond to a real increment of the optical aberrations in the older normal eye. This is because the other important effect, i.e., the intraocular scattering, practically does not affect our MTF's appreciably because we subtracted the scattering halo from the aerial image before we computed the MTF. The ratio of MTF's has a peak at intermediate spatial frequencies (Fig. 4), whereas the CSF ratios tend to increase monotonically with spatial frequency. One possible reason is that the CSF measurements can be influenced by chromatic aberrations or senile miosis (according to our clinical CSF data). In addition, intraocular scattering can be a factor increasing the CSF ratio for higher spatial frequencies.

This decline in retinal image contrast could explain a large fraction of the contrast-sensitivity loss found in older subjects. This is in agreement with recent interferometric measurements of contrast sensitivity, ${ }^{14}$ where quite similar neural CSF for younger and older subjects were found. This result also suggests a major role of the optical degradation in the spatial contrast-sensitivity decline with age. Important questions here are: What are the reasons for this deterioration in image quality, or, alternatively, why should ocular aberrations be larger in the older eye? With aging the lens becomes thicker and the refraction index and optical density increase, mainly in the nucleus, which could increase the aberrations. Also, any modification of the shape of the lens surfaces with age would affect aberrations. It would be interesting to evaluate the type and magnitude of aberrations that are modified with age. This could constitute a subsequent study in which the wave aberration for older eyes could be computed from the aerial retinal images presented above with the use of a phase-retrieval method. ${ }^{24}$

As was mentioned above, scattering in the ocular media increases with age, causing a large halo in the retinal image that would cause an additional reduction in image contrast. In this study we limited the effect of scattering by subtracting a background value (average in the four corners of the image) from the aerial images before we computed the MTF. If the scattering halo is approximately flat, then we remove most of its effect; otherwise there are some remaining effects of scattering that spread the aerial images farther apart from optical aberrations. As we have discussed elsewhere,$^{18}$ our experimental double-pass system in its present configuration is not capable of measuring intraocular scattering. We were able to detect some differences in the scattering halo of the aerial images of younger and older subjects, but our setup would need to be improved in its recording stage to produce additional accurate measurements of intraocular scattering. Because of our image-processing procedure, the decline in modulation observed in older subjects must be due primarily to an increase in optical aberrations exceeding intraocular scattering. Although we did not include intraocular scattering in our MTF calculations, following a traditional tendency resulting from the lack of a theoretical framework to consider aberrations and

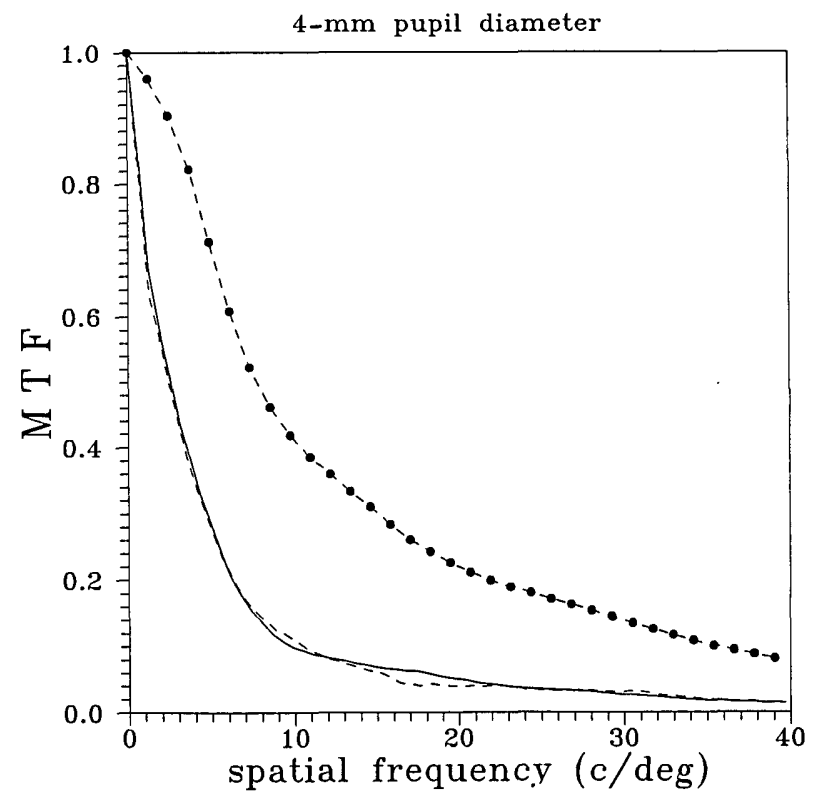

Fig. 7. Mean MTF for five older subjects implanted with monofocal IOL's (solid curve), MTF for subject MG's left eye (implanted with an IOL; dashed curve), and MTF for MG's right (normal) eye (dashed curve with filled symbols). 
scattering simultaneously, both factors together affect the retinal image under normal conditions.

The MTF's obtained in normal older subjects were very similar to those obtained in older subjects with implanted monofocal IOL's, which is in good agreement with previous CSF results in similar groups of subjects. ${ }^{15}$ One implication of this result is that the optical performance of a monofocal IOL would be approximately the same as that of the old lens, both performing worse than younger lenses. In addition, this leads us to conclude that the degradation of MTF's with age is due primarily to changes in the lens more than to changes in other ocular media. This is also in agreement with the results of Block and Rosenblum, ${ }^{25}$ who measured the MTF of the crystalline lens in vitro, finding a lower MTF for the aged lens.

Another factor that could affect double-pass MTF's differently in younger and older subjects is retinal reflection. We have assumed in previous work that the effect of retinal reflection on the double-pass MTF estimates is of little importance in younger subjects. However, this assumption also needs to be confirmed in the older eye. We must make certain that the decline in retinal image quality is not due largely to blurring of the aerial image in the retinal reflection. We assumed that the effect of the retinal reflection on the double-pass MTF would remain small with age. Some previous results also support this assumption. Gorrand ${ }^{26,27}$ showed that directionality in retinal reflection remains approximately constant with age, which could imply that the effect of retinal reflection on double pass would be similar in both younger and older subjects. We also performed another experiment, which addresses this question, by measuring MTF's in older and younger subjects with the same type of implanted monofocal IOL's. The results (see Fig. 7) show that MTF's are practically the same in younger and older subjects with implanted IOL's. This suggests that retinal reflection has a small, similar effect on MTF's obtained by the double-pass method in older and younger subjects.

In this research, subjects of intermediate ages ranging between 40 and 60 years were not considered. Hamilton et $a l .^{28}$ recently showed that different visual factors remain practically constant until the age of 50-60 years. If this is also true for retinal image quality, there must be a rapid increase of ocular aberrations at $\sim 50$ years of age, but this hypothesis must be further confirmed.

In conclusion, we studied the retinal image quality in the fovea for older subjects and showed a large increment of ocular aberration with age. In the future the doublepass method could be also used to study other optical characteristics of the aged eye, such as peripheral image quality, chromatic aberrations, and intraocular scattering.

\section{ACKNOWLEDGMENT}

This research was supported by the Comisión Interministerial de Ciencia y Tecnología, Spain, under grant TIC910438.

\section{REFERENCES}

1. R. A. Weale, The Aging Eye (H. K. Lewis, London, 1063).

2. C. Owsley and M. E. Sloane, "Vision and aging," in Handbook of Neuropsychology, F. Boller and J. Grafman, eds. (Elsevier, Amsterdam, 1990), Vol. 4, pp. 229-249.
3. J. S. Werner, D. H. Peterzell, and A. J. Scheetz, "Light, vision and aging," Optom. Vision Sci. 67, 2114-2229 (1990).

4. R. A. Weale, "Senile changes in visual acuity," Trans. Ophthalmol. Soc. 95, 36-38 (1975).

5. G. Derefeldt, G. Lenerstrand, and B. Lundh, "Age variations in normal human contrast sensitivity," Acta Ophthalmol. 57, 679-690 (1979).

6. C. Owsley, R. Sekuler, and D. Siemsen, "Contrast sensitivity throughout adulthood," Vision Res. 23, 689-699 (1983).

7. M. A. Johnson and D. Choy, "On the definition of age-related norms for visual function testing," Appl. Opt. 26, 1057-1063 (1987).

8. K. E. Higgins, M. J. Jaffe, R. C. Caruso, and F. M. de Monasterio, "Spatial contrast sensitivity: effects of age, testretest, and psychophysical method," J. Opt. Soc. Am. A 5, 2173-2180 (1988).

9. M. E. Sloane, C. Owsley, and C. Jackson, "Aging, senile miosis and spatial contrast sensitivity at low luminances," Vision Res. 28, 1235-1246 (1988).

10. A. Arnulf and O. Dupuy, "Le transmision des contrastes par le systeme optique de l'oeil et les seuils de contrastes retiniennes," C. R. Acad. Sci. 250, 2757-2759 (1960).

11. F. W. Campbell and D. G. Green, "Optical and retinal factors affecting visual resolution," J. Physiol. (London) 181, 575593 (1965).

12. M. Dressler and B. Rassow, "Neural contrast sensitivity measurements with a laser interference system for clinical screening and applications," Invest. Ophthalmol. Vis. Sci. 21 737-744 (1981).

13. D. B. Elliot, "Contrast sensitivity decline with aging: a neural or optical phenomenon?" Ophthalmol. Physiol. Opt. 7, 415-419 (1987).

14. K. B. Burton, C. Owsley, and M. E. Sloane, "Aging and neural spatial contrast sensitivity," Vision Res. (to be published).

15. C. Owsley, T. Gardner, R. Sekuler, and H. Lieberman, "Role of the crystalline lens in the spatial vision loss of the elderly," Invest. Ophthalmol. Vis. Sci. 26, 1165-1170 (1985).

16. J. Santamaría, P. Artal, and J. Bescós, "Determination of the point-spread function of the human eye using a hybrid optical-digital method," J. Opt. Soc. Am. A 4, 1109-1114 (1987).

17. R. Navarro, P. Artal, and D. R. Williams, "Modulation transfer of the human eye as a function of retinal eccentricity," J. Opt. Soc. Am. A 10, 201-212 (1993).

18. P. Artal and R. Navarro, "Simultaneous measurement of twopoint-spread functions at different locations across the human fovea," Appl. Opt. 31, 3646-3565 (1992).

19. A. Arnulf, J. Santamaría, and J. Bescós, "A cinematographic method for the dynamic study of the image formation by the human eye. Microfluctuations of the accommodation," J. Opt. 12, 123-128 (1981).

20. D. Sliney and M. Wolbarsht, Safety with Lasers and Other Optical Sources (Plenum, New York, 1980).

21. P. Artal, "Calculations of two-dimensional foveal retinal images in real eyes," J. Opt. Soc. Am. A 7, 1374-1381 (1990).

22. G. Walsh and W. N. Charman, "The effect of pupil centration and diameter on ocular performance," Vision Res. 28, 659665 (1988).

23. R. Navarro, M. Ferro, P. Artal, and I. Miranda, "Modulation transfer function of eyes implanted with intraocular lenses," Appl. Opt. (to be published).

24. P. Artal, J. Santamaría, and J. Bescós, "Retrieval of the wave aberration from actual point-spread function data," J. Opt. Soc. Am. A 5, 1201-1206 (1988).

25. M. G. Block and W. M. Rosenblum, "MTF measurements on the human crystalline lens," J. Opt. Soc. Am. A 4, P7 (1987).

26. J. M. Gorrand, "Reflection characteristics of the human fovea assessed by reflecto-modulometry," Ophthalmol. Physiol. Opt. 9, 53-60 (1989).

27. J. M. Gorrand, Universite de Clermont-Ferrand, Clermont, France (personal communication).

28. J. M. Harrison, R. A. Appelgate, J. T. Yates, and C. Ballentine, "Contrast sensitivity, glare susceptibility and age in the range from 20-50 years old," Invest. Ophthalmol. Vis. Sci. Suppl. 33, 1380 (1992). 